\title{
Letter to Editor: Alarming Level of Tramadol Intoxication in Iran
}

\author{
Mohammad Delirrad $^{1}$, Ali Banagozar Mohammadi ${ }^{*}$ Q \\ 1. Department of Forensic Medicine and Toxicology, School of Medicine, Urmia University of Medical Sciences, Urmia, Iran. \\ 2. Department of Internal Medicine, Faculty of Medicine, Tabriz University of Medical Sciences, Tabriz, Iran.
}

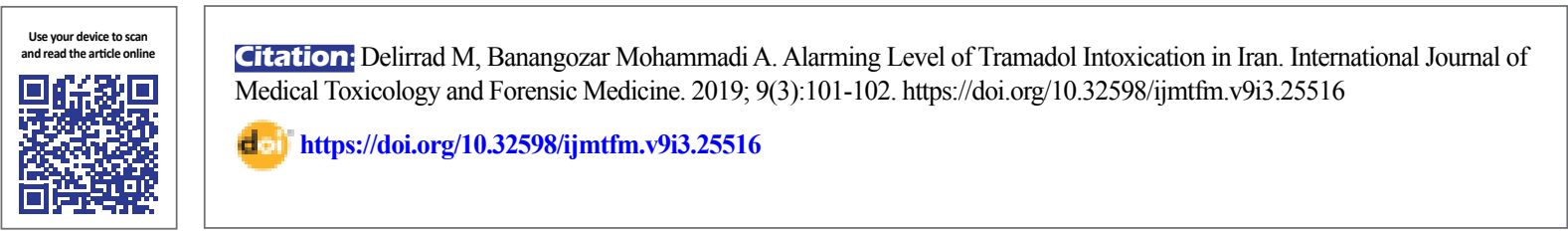

\section{7 he use of tramadol, as a medicine, has be- gun in Iran since 15 years ago and it is in- creasingly abused nowadays.}

As clinical toxicologists in two distinctive referral hospitals in the northwest of Iran, we have been visiting many patients with tramadol overdose. The authors have also completed a clinical toxicology fellowship program in Mashhad (The northeast of Iran) for 1.5 years. Moreover, by obtaining information from other colleagues in different regions of Iran, we realized that a similar pattern of tramadol abuse and complications exist all around the country.

Observing patients with tramadol-induced seizures is a part of our daily practice. In the past, we had several patients with life-threatening complications following tramadol overdose. For example, a patient, who had tramadol overdose (20 grams), deceased following recurrent convulsions. Another patient, who had ingested $2 \mathrm{~g}$ tramadol, had status epilepticus on admission resulting in hypoxic-ischemic encephalopathy and, finally, persistent vegetative state.

Other complications in our patients included the decreased levels of self-awareness and apnea (especially when tramadol was co-ingested with other sedativehypnotics or alcohol), serotonin toxicity, rhabdomyolysis, electrolyte disturbances, renal failure, QT interval prolongation, cardiac dysrhythmias, and various traumas (even life-threatening) during convulsive seizures such as skull fractures, intracranial hemorrhages, jaw dislocations, shoulder dislocations (unilateral or even bilateral), chest or abdominal injuries, and withdrawal syndrome because of the discontinuation or reduction of tramadol dose.

Several years ago, the importance of these complications was unclear for us. Therefore, we did not report the cases. Over time, by encountering the increasing number of patients with tramadol intoxication, the situation became more evident. By reviewing the literature, we found that only some of these side effects and complications of tramadol were implicitly mentioned in toxicology textbooks $[1,2]$. Also, by reviewing the valid academic databases such as PubMed, Scopus, and Web of Science, we noticed a few reports about the complications of tramadol, such as convulsions, serotonin syndrome, or rhabdomyolysis in the literature. Besides, many of the published articles were either case reports or letters to the editor except 1 case series [3].

More recently, some interesting articles have been published by Journal of the American Medical Association (JAMA) and Lancet authoritative journals concerning "increased mortality in patients with tramadol use comparing other analgesics", "increased tramadol intoxication and complications following excessive and inappropriate administration of tramadol", or "an increasing

* Corresponding Author:

Ali Banagozar Mohammadi, $M D$.

Address: Department of Internal Medicine, Faculty of Medicine, Tabriz University of Medical Sciences, Tabriz, Iran.

Tel: +98 (41) 35498260

E-mail: alibanagozar@gmail.com 
trend in the prescription of tramadol for patients with osteoarthritis or postoperative pain" [4-7]. In these reports, the main reason for the increasing prescription of tramadol in the developed countries was the recommendations of pain control guidelines [4-7].

In Iran, several hypotheses may be considered for high rate of tramadol complications; they include 1 . The abuse and misuse of the drug by Iranians; 2 . The possibility of unauthorized access to tramadol through some pharmacies; 3 . The falsification of tramadol available in the market and 4. Deceptive advertising about tramadol effects on sexual strength or treatment of obesity or body making.

Although tramadol was prescribed by doctors for pain control, it is easily accessible (in original or counterfeit forms) for people, who are seeking it. Domestic pharmaceutical manufacturers produce $50 \mathrm{mg}$ and $100 \mathrm{mg}$ oral forms of tramadol, but other forms $(200 \mathrm{mg}, 225 \mathrm{mg}$, $300 \mathrm{mg}$, $400 \mathrm{mg}$, and $500 \mathrm{mg}$ tablets) are produced either legally (by some pharmaceutical companies in other countries) or illegally (in clandestine laboratories inside or outside of Iran). Little information exists about the active ingredients and compounds of these illegally-presented drugs. There is also misleading advertising on the effects of tramadol on sexual strength, the treatment of impotency, or obesity. Therefore, the drug is more likely to be abused by adolescents and adult men. Nonetheless, in scientific resources, tramadol is recommended only for certain types of sexual dysfunction (premature ejaculation) [8]. Most women use tramadol for the treatment of obesity or suicidal attempts.

Although tramadol abuse and its complications in Iranian society have reached an alarming situation, the investigations in this filed are not proper and adequate. Therefore, toxicologists, pharmacologists, and other clinicians are recommended to perform further studies concerning tramadol toxicity and its complications; meanwhile, determining the compounds presented in the counterfeit forms of tramadol supplied in Iran and their complications is essential.

\section{Ethical Considerations}

\section{Compliance with ethical guidelines}

There was no ethical considerations to be considered in this letter.

\section{Funding}

This letter did not receive any specific grant from funding agencies in the public, commercial, or not-for-profit sectors.

\section{Author's contributions}

Conceptualization: Ali Banagozar Mohammadi; Methodology, investigation, writing-original draft: Mohammad Delirrad; Writing-review \& editing, resources, supervision: Ali Banagozar Mohammadi.

\section{Conflict of interest}

The authors declared no conflict of interest.

\section{References}

[1] Howland MA, Lewin NA, Nelson LS, Goldfrank LR, Hoffman RS. Goldfrank's Toxicologic Emergencies, $10^{\text {th }}$ edition. Pennsylvania: McGraw-Hill Education; 2014

[2] Walls R, Hockberger R, Gausche-Hill M. Rosen's emergency medicine: Concepts and clinical practice, $9^{\text {th }}$ edition. Amsterdam: Elsevier Health Sciences; 2017.

[3] Tashakori A, Afshari R. Tramadol overdose as a cause of serotonin syndrome: A case series. Clinical toxicology (Philadelphia). 2010; 48(4):337-41. [DOI:10.3109/15563651003709427]

[4] Zeng C, Dubreuil M, LaRochelle MR, Lu N, Wei J, Choi $\mathrm{HK}$, et al. Association of tramadol with all-cause mortality among patients with osteoarthritis. Jama. 2019; 321(10):969-82. [DOI:10.1001/jama.2019.1347]

[5] Glare P, Aubrey KR, Myles PS. Transition from acute to chronic pain after surgery. The Lancet. 2019; 393(10180):1537-46. [DOI:10.1016/S0140-6736(19)30352-6]

[6] Neuman MD, Bateman BT, Wunsch H. Inappropriate opioid prescription after surgery. The Lancet. 2019; 393(10180):1547-57. [DOI:10.1016/S0140-6736(19)30428-3]

[7] Colvin LA, Bull F, Hales TG. Perioperative opioid analgesiawhen is enough too much? A review of opioid-induced tolerance and hyperalgesia. The Lancet. 2019; 393(10180):1558-68. [DOI:10.1016/S0140-6736(19)30430-1]

[8] Martyn-St James M, Cooper K, Kaltenthaler E, Dickinson K, Cantrell A, Wylie K, et al. Tramadol for premature ejaculation: A systematic review and meta-analysis. BMC Urology. 2015; 15:6. [DOI:10.1186/1471-2490-15-6] 